\title{
Want to read more?
}

\section{Acupuncture}

- Acupuncture is often advocated as adjunct treatment during stroke rehabilitation. This meta-analysis found that acupuncture treatment did not improve function for patients after stroke (see www.cmaj.ca/content /182/16/1723.full).

- In a unique subtype of acupuncture known as Hari, needles are permanently placed into subcutaneous tis-

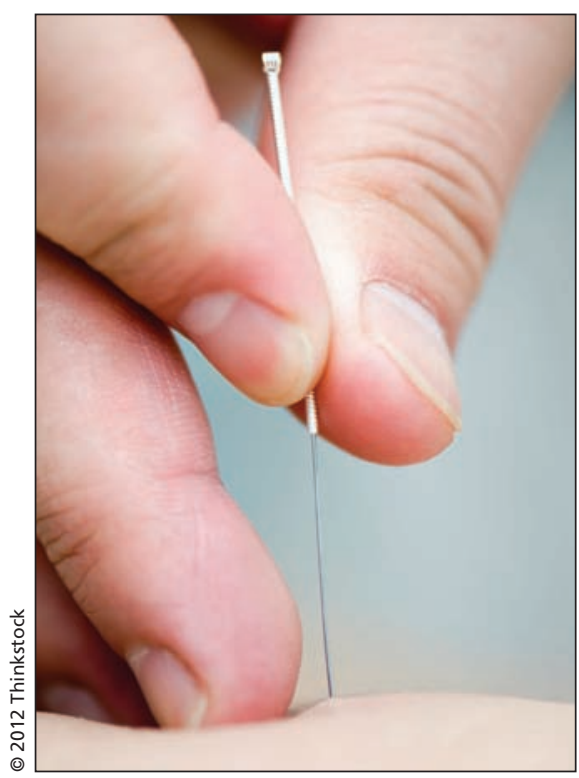

sues along predefined meridiens. The relative safety, compared with traditional acupuncture, is unknown (see www.cmaj.ca/content/183/13 /E1038.full).

\section{Migraine}

- Migraine prophylaxis should be considered for patients whose headaches affect their quality of life despite appropriate symptomatic treatment and for those who are at risk of medication-overuse headache. Pringsheim and colleagues address common questions around medication choice and knowing when to start and stop prophylaxis (see www.cmaj .ca/content/182/7/E269).

- Sometimes migraine medication can have unexpected adverse effects. This 29-year-old man receiving antiretroviral therapy for HIV developed an ancient complication called "Holy Fire," after a severe migraine was treated with ergotamine tartrate (see www.cmaj .ca/content/182/4/378.full).

\section{Tattoo reactions}

- Three weeks after a 30-year-old man received a tattoo, the appearance of the tattoo changed dramatically, becoming three-dimensional. Molluscum contagiosum, most likely inoculated by a contaminated needle, was implicated (see www.cmaj.ca/content /182/9/E382.full).

- Black henna tattoos generally last one to two weeks on the skin, but they can have longer-lasting effects. Exposure to high concentrations of the additive, paraphenylendiamine (PPD), often found in henna mixtures, can cause potent skin sensitization. Subsequent exposure to PPD in hair dyes and rubber products can result in acute contact dermatitis (see www.cmaj.ca/content/176/4/445.full).

\section{Exercise-induced rhabdomyolysis}

- Although rhabdomyolysis induced by exercise is typically seen in athletes involved in high-endurance activities, it can occur after shorter intense work-outs. Kiberd and Campbell stress that aggressive intravascular volume expansion in rhabdomyolysis can reduce the likelihood of kidney damage (see www.cmaj.ca /content/183/16/E1222).

CMAJ 2012. DOI:10.1503/cmaj.120250

\section{More Practice online}

\section{Radiation dose and protection}

Although computed tomography is a useful tool in many clinical situations, it delivers a higher dose of radiation compared with some other imaging modalities. Use of alternative imaging techniques, such as ultrasound and magnetic resonance imaging, may be more appropriate for some conditions. M.O. Baerlocher, S. Leung, M. Asch, A. Myers

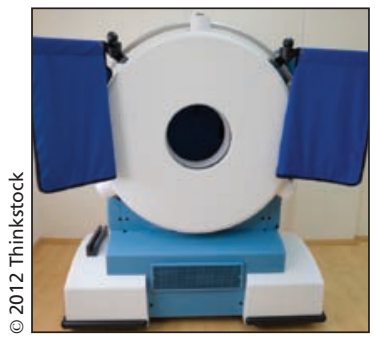

\section{Lingua plicata}

Deep grooves or fissures on the dorsal and lateral surfaces of the tongue can be caused by a benign disorder called lingua plicata. Most common in older people, it can result in halitosis and focal glossitis if food accumulates in the fissures. Good oral hygiene with brushing of the tongue can reduce complications. - L. Nisa, R. Giger

For more Practice articles, see www.cmaj.ca/site/home /practice.xhtml

CMAJ 2012. DOI:10.1503/cmaj.120239 